\title{
L'imprécation de Clytemnestre. Véhémence et performance sur la scène racinienne
}

\section{Gilles Declercq}

\section{(2) OpenEdition}

1 Journals

Édition électronique

URL : http://journals.openedition.org/rhetorique/99

DOI : $10.4000 /$ rhetorique. 99

ISSN : 2270-6909

Éditeur

UGA Éditions/Université Grenoble Alpes

Édition imprimée

ISBN : 978-2-84310-255-4

\section{Référence électronique}

Gilles Declercq, «L'imprécation de Clytemnestre. Véhémence et performance sur la scène racinienne ", Exercices de rhétorique [En ligne], 1 | 2013, mis en ligne le 18 juin 2014, consulté le 12 septembre 2020. URL : http://journals.openedition.org/rhetorique/99 ; DOI : https://doi.org/10.4000/rhetorique. 99

Ce document a été généré automatiquement le 12 septembre 2020.

\section{(c) () (ㅇ)}

Les contenus de la revue Exercices de rhétorique sont mis à disposition selon les termes de la Licence Creative Commons Attribution - Pas d'Utilisation Commerciale - Partage dans les Mêmes Conditions 4.0 International. 


\title{
L'imprécation de Clytemnestre. Véhémence et performance sur la scène racinienne
}

\author{
Gilles Declercq
}

1 La présente étude croise deux axes de notre recherche sur la rhétorique et le théâtre de Racine $^{1}$. Le premier relève de la rhétorique des passions, appréhendée ici sous la forme extrême d'un discours de malédiction (Iphigénie, V,4). Le second porte sur la conjonction, problématique, de l'imaginaire discursif (que suscitent les grandes figures de vision et d'interpellation in absentia: apostrophe, prosopopée, hypotypose) et de l'image scénique du corps actorial, figure in praesentia du personnage furieux.

2 Notre propos s'inscrit ainsi dans une réflexion sur la véhémence entendue comme modalité intense, voire paroxystique, de représentation des passions. Notre objet d'étude est la représentation de l'intensité passionnelle sur la scène racinienne - au plan dramaturgique, rhétorique et esthétique : la représentation des grandes passions et le grand style que leur assigne corollairement la rhétorique depuis Cicéron². Mais il s'agit aussi d'identifier le spectacle de la passion et sa construction sur scène dans son rapport concret-visuel, auditif-au spectateur. Notre perspective rhétorique s'articule ainsi à la problématique théâtrale de la performance : discerner les possibles de la représentation tels que le texte, c'est-à-dire le «script » de théâtre, les suggère voire les programme. Nous n'adopterons donc pas la définition soustractive que Roland Barthes proposait de la théâtralité : «le théâtre moins le texte, [...] une épaisseur de signes et de sensations qui s'édifie sur la scène ${ }^{3}$. " Nous nous focaliserons au contraire sur la dynamique vectorielle qui lie le texte à sa performance, comme le discours actualise le texte en rhétorique. Car c'est précisément cette perspective qui définit l'écriture théâtrale au xvII ${ }^{\mathrm{e}}$ siècle où la représentation prime sur l'édition du texte: Racine n'écrit pas au sens absolu et moderne de ce terme, mais compose, comme poète dramatique, pour être représenté, déclamé et incarné ${ }^{4}$; le travail éditorial est ultérieur et second. La quête de notoriété qui anime le théâtre de l'âge classique procède d'abord 
de la conquête de la scène et des spectateurs, comme en témoigne la carrière de Jean Racine.

3 Cette double perspective, rhétorique et scénique, requiert de définir quelques termes clés pour cette étude :

a) Dramaticité s'entendra au sens aristotélicien du "système des faits", principe de concaténation causal des événements (sunthesis tôn pragmatôn) qui convertit la fable en drame ${ }^{5}$. La dramaticité détermine la nécessité de l'action; elle constitue pour Aristote la fin de la tragédie, la peinture des caractères n'étant qu'incidente à l'avancée de l'action ${ }^{6}$.

b) Théâtralité s'entendra consécutivement par opposition à dramaticité. Celle-ci est relative à l'action ; celle-là, au regard. La dramaticité est une problématique structurale intrascénique. Inversement, la théâtralité est d'ordre extrascénique ; elle a pour fin et origine le spectateur tel que le dispositif théâtral le constitue en déterminant l'étendue et la modalité de son regard. Et la notion de dispositif théâtral en tant qu'elle implique une intentionnalité, permet de différencier théâtralité et spectacularité :

1. La théâtralité en tant que dispositif de structuration du regard excède le cadre générique de la dramaticité : elle définit globalement la modalité de réception de ce qui est construit et donné à regarder. La théâtralité est une catégorie transgénérique, qui hante notamment la rhétorique chaque fois que celle-ci met en œuvre la composante visuelle du discours pour capter l'attention de l'auditoire.

2. La théâtralité se distingue encore de la spectacularité, par la nature stratégique et intentionnelle de son dispositif qui concerne non pas l'objet à voir (le regardé) mais le sujet $d u$ voir (le regardant). Si la spectacularité, en tant que le monde visible s'offre au regard, peut être remarquable, voire fascinante, elle est toujours accidentelle et dépourvue d'intention. Inversement, la théâtralité ne se contente pas d'attirer le regard, mais le structure. Toujours intentionnelle, elle procède du calcul rationnel: une intention esthétique et/ou critique la fonde ${ }^{7}$. Par cette emprise sur le spectateur, la théâtralité s'apparente au pouvoir de la rhétorique sur l'auditeur. Réflexion structurée du monde, elle établit par le regard qu'elle construit, le lien esthétique et cognitif du théâtre à la philosophie.

6 c) Rhétoricité, enfin, s'entendra au sens fondamental que lui donne Aristote dans la Rhétorique - à savoir la recherche spéculative de tous les modes du persuasif pour une situation donnée ${ }^{8}$. La rhétoricité définit la visée persuasive inhérente à tout discours, comme le formule Bernard Lamy en posant l'équivalence entre art de parler et art de persuader:

Nous ne parlons que pour faire entrer dans nos sentiments ceux qui nous écoutent : c'est pourquoi quand on dit l'Art de Parler, on fait connaître que la fin de cet Art est de Persuader, puisque c'est l'intention qu'ont tous ceux qui s'appliquent à bien parler 9 .

Dynamique vectorielle, la rhétoricité traverse donc le double discours théâtral : celui du discours dramatique, défini par les entreprises argumentatives que les personnages exercent les uns sur les autres au sein de l'univers intrascénique; celui du discours théâtral, entendu comme l'emprise extrascénique que l'œuvre établit ou cherche à établir sur le spectateur ${ }^{10}$.

7 Dans cette seconde perspective, la rhétoricité persiste même lorsque la rhétorique intrascénique est suspendue ou arrêtée : il y a toujours recherche d'un effet sur le spectateur. Cette rémanence lie rhétoricité et théâtralité, quand argumentation intrascénique et dramaticité font défaut. Or ce défaut caractérise précisément la scène 
de l'imprécation de Clytemnestre (Iphigénie, $\mathrm{V}, 4$ ) : discours de malédiction prononcé au seuil du dénouement, en raison du statut dramaturgique de cette scène qui se caractérise par la mise en panne de la dramaticité et la promotion corrélative de la théâtralité et de la rhétoricité extrascénique. Une scène à ce titre exemplaire de la manière dont la tragédie racinienne prédétermine et construit le regard du spectateur sur les passions ${ }^{11}$.

\section{Une scène inutile : théâtralité vs dramaticité}

8 L'analyse dramaturgique révèle en effet un statut hors-norme au regard des prescriptions aristotéliciennes: la scène n'est pas nécessaire, car elle n'est cause d'aucune scène ultérieure. Elle n'est pas régie par la logique de l'agencement des faits et se caractérise au contraire par la suspension de l'action scénique. L'acte antérieur, en effet, s'est conclu par la décision d'Ériphile de dénoncer le projet de fuite d'Iphigénie ; Ériphile quitte alors la scène pour rejoindre Calchas et assurer le sacrifice. Dans la scène immédiatement antérieure $(\mathrm{V}, 3)$, Iphigénie consent à son sacrifice et quitte à son tour la scène, des gardes armés empêchant Clytemnestre de quitter la scène. L'action, au sens précis de l'avancée du drame, s'accomplit donc désormais hors-scène - ce qui signifie inversement que la scène est vide d'action. La scène $V, 4$ est donc littéralement a-dramatique et son existence contredit la doxa qui veut que la dramaturgie classique, a fortiori la dramaturgie racinienne tenue pour son paradigme, soit toute régie par la logique de l'action nécessaire et de l'enchaînement causal. Il n'en est rien et nous avons ici le contre-exemple d'une scène inutile, degré zéro de la dramaticité.

9 Le corrélat rhétorique de cette a-dramaticité est l'aporie du genre délibératif, genre recteur de la tragédie. Ce statut recteur découle de la définition cornélienne de la tragédie : drame mettant en péril la Cité ou son Prince ${ }^{12}$. Genre essentiellement politique, la tragédie régulière est consécutivement régie par le mode rhétorique énonçant les décisions des protagonistes princiers; décisions qui, conformément au principe de causalité liant les actes aux discours sur la scène classique ${ }^{13}$, déterminent l'avancée du drame vers sa fin ${ }^{14}$. Inversement, dans notre scène, la situation est caractérisée par la paralysie d'action et de décision, la double impuissance d'une mère et reine ${ }^{15}$ :

\section{CLYTEMNESTRE :}

Ah! vous n'irez pas seule, et je ne prétends pas...

Mais on se jette en foule au-devant de mes pas.

Perfides, contentez votre soif sanguinaire.

AEGINE :

Où courez-vous, Madame ? Et que voulez-vous faire? [1668-1670]

Clytemnestre ne peut infléchir la décision de sa fille, et elle est repoussée par les gardes qui demeurent sur scène. Son impuissance physique se traduit alors par une gesticulation scénique et, corrélativement, l'aporie délibérative frappe son discours. Spectatrice forcée d'une action qui lui échappe, et dont le dire n'infléchit plus le drame, Clytemnestre cherche querelle. Proférant accusation et malédiction, elle investit par son discours les genres judiciaire et épidictique.

11 L'étude de cette gesticulation et de cette inflexion - qui définissent la véhémence de Clytemnestre - impose deux remarques liminaires.

12 1) La mise en abîme du genre épidictique : l'effondrement de la parole délibérative a pour conséquence la promotion des genres judiciaire et épidictique; une altération qu'il 
convient d'évaluer en la confrontant au régime ordinaire des genres oratoires, et en distinguant les deux régimes rhétoriques ordonnant la représentation: celui de la rhétorique intrascénique (interactions argumentatives des personnages); celui de la rhétorique extrascénique (effet de la représentation sur le spectateur) :

- En rhétorique intrascénique, le genre délibératif est recteur; les genres épidictique ou judiciaire constituent des sous-genres fonctionnellement subordonnés à la parole délibérative laquelle, en tant que parole active, assure l'avancée du drame. L'apparition intrascénique du judiciaire ou de l'épidictique est donc à lire comme symptôme et effet de l'enrayement du drame.

- En rhétorique extrascénique, c'est inversement l'épidictique qui constitue le genre recteur ordinaire. Cible des arts de plaire parmi lesquels le théâtre se range explicitement, le spectateur de l'âge classique est l'objet d'une visée esthétique, qui définit l'auditoire de l'éloquence épidictique. Ce n'est qu'à partir de cette réception esthétique première que le spectateur peut, le cas échéant, tirer leçon du spectacle dans l'ordre du délibératif ou du judiciaire.

La répartition des genres se présente donc ainsi :

\begin{tabular}{|l|l|}
\hline Rhétorique intrascénique & Rhétorique extrascénique \\
\hline $\begin{array}{l}\text { genre recteur macrostructural } \\
\text { délibératif } \\
\text { (induit l'action) }\end{array}$ & $\begin{array}{l}\text { genre recteur macrostructural } \\
\text { éidictique } \\
\text { (induit le plaisir du spectateur) }\end{array}$ \\
\hline $\begin{array}{l}\text { genres secondaires microstructuraux } \\
\text { judiciaire, épidictique } \\
\text { (subordonnés à la prise de décision } \\
\text { et à l'avancée de l'action) }\end{array}$ & $\begin{array}{l}\text { genres secondaires microstructuraux } \\
\text { délibératif;judiciaire } \\
\text { (subordonnés à l'effet esthétique } \\
\text { de la représentation sur le spectateur) }\end{array}$ \\
\hline
\end{tabular}

14 La particularité de notre scène est la promotion intrascénique des genres oratoires secondaires, conséquence de l'aporie délibérative. Avec la malédiction de Clytemnestre, c'est plus spécifiquement le genre épidictique qui vient redoubler au plan intrascénique le genre recteur extrascénique, créant un dispositif remarquable de mise en abîme des regards : le spectateur est invité à regarder le regard que Clytemnestre porte sur ellemême et sur la catastrophe tragique. La scène est ainsi régie par une esthétique spéculaire qui fait la singularité de sa théâtralité.

2) La monstration des passions : l'a-dramaticité promeut la théâtralité: la scène ne donnant pas à faire, donne à voir et se donne à voir. Tel est le sens et la fonction des premiers vers qui désignent l'impuissance de la reine et son agitation vaine (« où courez-vous, Madame»), et annoncent un spectacle que le public est appelé à évaluer selon le code épidictique - la représentation véhémente et sublime des passions. Sublime non pas au sens longinien, mais au sens cicéronien de l'oratio grandis; passions au sens littéral d'émotions vaines, privées d'efficacité dramatique, mais objet en revanche d'une monstration théâtrale dont le spectateur est convié à apprécier l'intensité.

16 En conséquence ce préambule est fonctionnellement métadiscursif. Il ne s'agit pas de mettre en œuvre une fureur agissante (telle la fureur de Thésée contre Hippolyte) ; mais 
de mettre en scène une fureur impuissante. L'objet rhétorique et théâtral de la scène n'est pas le discours passionné (qui exprime les passions), mais le discours passionnel (qui les mentionne et les soumet à l'analyse du regard ${ }^{16}$ ). La scène ne relève donc pas $d u$ mode simple de l'éloquence passionnelle; elle procède du régime métadiscursif de la rhétorique des passions : elle est spectacle annoncé d'une fureur véhémente.

\section{Corps et Paroles en véhémence}

Nous voudrions à présent caractériser ce dispositif théâtral, par un triple questionnement : 1) la question du corps racinien dans son rapport au «trouble» passionnel ; 2) la question actoriale de la parole de malédiction; 3) la question du rapport de l'imprécation (qui se déploie selon l'imaginaire de la cosmologie antique) au présent de la performance et de l'image scéniques.

\section{La double figure du corps racinien}

Il suffit ici, pour invalider la doxa qui veut que le corps racinien soit abstrait, de souligner contradictoirement l'ensemble des signes qui réfèrent au corps de l'acteur, pour peu qu'on prête attention aux didascalies internes du texte racinien. Dans ce "script» destiné à être joué et incarné, les trois premiers vers désignent et programment les mouvements de l'actrice incarnant Clytemnestre: avancée vers les gardes à la poursuite d'Iphigénie qui s'éloigne ( $\mathrm{Ah}$ ! vous n'irez pas seule, et je ne prétends pas...»); arrêt brutal, souligné scripturairement par la suspension, et scéniquement marqué par l'interposition physique des gardes (« Mais on se jette en foule au-devant de mes pas »); puis nouvel élan, potentiellement suicidaire, vers les armes des gardes - Clytemnestre s'exposant aux coups, comme plus tard Phèdre à l'épée d'Hippolyte («Perfides, contentez votre soif sanguinaire»). Quant au vers suivant d'Aegine (« Où courez-vous, Madame ? et que voulez-vous faire »), il désigne un mouvement d'oscillation stérile sur le plateau : de l'avant-scène lumineuse à l'arrièrescène obscure comme y prédisposait le rectangle en profondeur des premières scènes du XvII ${ }^{e}$ siècle, ou de cour à jardin selon le rectangle en largeur de nos scènes modernes, ou encore en déambulation circulaire, irritée et harcelante, figure fréquente dans les mises en scène d'Antoine Vite $z^{17}$. Autant de déplacements et de mouvements possibles, que suggèrent ces vers liminaires, véritable préambule scénique.

Référant simultanément au corps scénique de l'acteur et au corps symbolique du personnage, la réplique suivante de la reine est une figuration du trouble qui, dans une conjonction propre au langage classique, articule la valeur concrète et abstraite de la parole théâtrale :

Hélas ! je me consume en impuissants efforts;

Et rentre au trouble affreux, dont à peine je sors.

Mourrai-je tant de fois, sans sortir de la vie ? [1671-1673]

Dans le strict prolongement de l'actio des vers précédents, Clytemnestre offre la figuration scénique de son impuissance, selon la dynamique paradoxale d'une energeia vainement dépensée :

- En premier lieu, antithèse et oxymore structurent ce groupe de vers : les "efforts » sont « impuissants »; la « rentrée » annule la « sortie » du trouble ; la mort itérative est incapable 
de mettre un terme à une vie de tourments. La forme verbale de ces contradictions aporétiques traduit l'agitation stérile d'un corps privé de toute échappée.

- En second lieu, la course vaine cessant, le corps de la reine se fige et se replie sur lui-même dans une torsion déchirante, immobilité tourmentée, lexicalement désignée comme trouble. Or le trouble en question est d'abord une figure physique offerte sur la scène au spectateur, et dont les vers prononcés par Clytemnestre constituent l'ekphrasis. Il faut en effet se prémunir de l'inversion de sens qui découle de la lecture du texte, en aval de sa représentation, et qui tend à donner à ces vers une valeur exclusivement «figurée ", morale ou psychique. En représentation c'est-à-dire in praesentia, le trouble n'est pas l'essence abstraite de la passion éprouvée par Clytemnestre, mais le signe concret d'une agitation tourbillonnaire conforme à la logique scénique de la monstration des passions.

- Certes, cette agitation figure aussi le tourment psychique de Clytemnestre, déchirée entre impuissance et fureur. Mais cette figuration psychique ne substitue pas un sens abstrait à la figure concrète du corps. La visualité du trouble est sa donnée première, théâtrale et scénique; une visualité toutefois bivalente, car elle procède du statut du visible au XvII siècle où le signe est encore déterminé par l'herméneutique allégorique qui régit les arts du spectacle renaissants et classiques ${ }^{18}$. Or le signe allégorique est polysémique et doté d'un envers et d'un avers articulant valeur concrète et abstraite, univers corporel et spirituel ${ }^{19}$. Le corps troublé de Clytemnestre relève de cette phénoménologie - véritable pli sur pli, où le corps actorial, figure réfléchissante, fait transparaître l'âme du personnage, son éthos et ses passions :

Le grand air et l'apparence noble, l'humilité et l'apparence servile, la modération et la juste mesure, l'excès (hubris) ainsi que ce qui n'a aucune idée de la beauté (apeironkalon), c'est grâce au visage (prosopon) et c'est au travers des attitudes (schèmatôn) dont les hommes usent dans leur façon de se tenir et de se mouvoir que cela transparaît (diaphainei) ${ }^{20}$.

Le propos est littéralement transposable à la «façon de se tenir et de se mouvoir » de Clytemnestre. Et au cœur de sa réplique, le vers, « [Je] rentre au trouble affreux, dont à peine je sors", serait hermétique si ne venait précisément l'éclairer la liaison allégorique des sens concret et abstrait. D'une part, l'avers spatial et scénique du plateau où s'agite et se prostre le corps de l'acteur ; celui du lieu fictionnel aussi, huis clos d'une tente qui enferme la reine. D'autre part, l'envers moral et spirituel, par l'adjonction du troisième vers («Mourrai-je tant de fois, sans sortir de la vie»), transposition métaphorique du « sortir / rentrer » en un " mourir / renaître », et où la mort au figuré désigne l'agonie, c'est-à-dire un «souffrir » infini. La clé herméneutique prend ici la forme d'un topos familier, celui du supplice infernal dont la figure allégorique de la reine - corps prostré, âme tourmentée - est le chiffre.

Une posture toute en tension qui est encore porteuse de l'expansion oratoire de la scène, puisque le filage de ce topos infernal va très exactement permettre l'amplification épidictique de la malédiction.

\section{Théâtre de la parole : l'épidictique noir}

Ô Monstre que Mégère en ces flancs a porté !

Monstre ! que dans nos bras les Enfers ont jeté.

Quoi tu ne mourras point ? Quoi pour punir son crime... [1679-1681]

La tirade de Clytemnestre succède à sa gesticulation; la dynamique cinétique fait place à la dynamique discursive. Mais l'impuissance de l'une et de l'autre est un invariant : la tirade véhémente accomplit le spectacle annoncé de la fureur... et vérifie son 
impuissance dramatique. La véhémence se définit donc comme intensité oratoire non performative : Clytemnestre accuse, condamne, maudit, mais sans avoir prise sur l'action en cours ni emprise sur les personnages qu'elle apostrophe.

Théâtralité sans efficace, la véhémence n'est pas la violence, mais son mime oratoire, très exactement son simulacre. L'imprécation de Clytemnestre est un morceau de bravoure oratoire, "spectaculaire » au sens ordinaire de ce terme : une représentation passionnelle, dont l'intensité est disproportionnée à son effet, dramatiquement nul. Exacerbant le lien du signe oratoire et théâtral au simulacre, la véhémence assume ainsi une fonction implicitement métathéâtrale. Mettant en jeu et en question la puissance du théâtre et de ses signes, sa rhétoricité éprouve ses limites en exacerbant le divorce entre théâtralité et dramaticité; et elle reverse toute l'efficacité de la représentation dans une rhétorique épidictique extrascénique, monstration des passions directement adressée au spectateur. Le regard de ce dernier est en ce sens un méta-regard: focalisé sur la construction du spectacle de la fureur et sa modalité véhémente, il se constitue en une analytique des passions.

Le premier effet de cette analytique est de mettre à mal la doxa critique qui veut que le discours passionnel distingue le protagoniste de son confident, personnage de l'humanité ordinaire étranger à la fureur. Or la fureur de Clytemnestre ne surgit pas de son âme enflammée, ni d'une "essence " tragique privilégiée. Le discours furieux procède au contraire du dialogue entre la Reine et Aegine, laquelle, en révélant la trahison d'Ériphile, fait fonction de détonateur illocutoire - acte de discours dont l'effet est l'irruption de la fureur, le surgissement de l'indignatio. Le propos de la confidente, tant par sa forme que par son effet, contribue donc de manière essentielle à la construction de la fureur. Littéralement, Aegine co-produit le discours passionnel :

Ah! Savez-vous le crime, et qui vous a trahie,

Madame? Savez-vous quel Serpent inhumain

Iphigénie avait retiré dans son sein?

Ériphile en ces lieux par vous-même conduite,

A seule à tous les Grecs révélé votre fuite. [1674-1678]

Cette coopération oratoire est attestée par l'elocutio imprécatoire, dont Aegine prend l'initiative par le topos mythologique du serpent. Combiné en l'occurrence au sème judéo-chrétien de la perfidie, le topos antique réfère aux Érinyes à la tête entourée de serpents. Topique que Clytemnestre amplifiera en évoquant Mégère, Érinye allégorique de la haine (v. 1679) : l'invective "serpent inhumain " réunit ainsi allégoriquement tératologie zoologique et monstruosité éthique.

Ce lieu de la monstruosité est encore l'index lexical de la théâtralité véhémente. Tel est le sens et la fonction de la seconde occurrence du terme, en attaque du vers :

ô Monstre, que Mégère en ces flancs a porté !

Monstre ! que dans nos bras les Enfers ont jeté. [1679-1680]

Par sa position prosodique et oratoire, le terme prend un sens absolu et une valeur métathéâtrale de monstration: "dans nos bras » réfère en effet non plus métaphoriquement à la fille de Mégère, mais à Ériphile, qui a effectivement bénéficié de l'hospitalité d'Iphigénie et de sa mère. "Monstre » fonctionne donc comme déictique, et sa référentialité permet à Clytemnestre d'apostropher Ériphile en lui signifiant une sentence de mort (« Quoi tu ne mourras point ? Quoi pour punir son crime... », v. 1681). Or cette logique punitive prend source dans la réplique d'Aegine ( $\mathrm{Ah}$ savez-vous le crime et qui vous a trahie?»,v.1674) qui fournit simultanément l'encodage 
mythologique du crimen et la mobilisation corrélative du genre judiciaire, aussitôt filé par Clytemnestre (envisageant le supplice d'Ériphile, la reine renverse la figure et se fait Érinye vengeresse). Cependant, l'universalisation et l'amplification du schème de la vengeance déplacent rapidement la tirade du genre judiciaire (accusatio) vers le genre épidictique (imprecatio) :

Mais où va ma douleur chercher une Victime?

Quoi pour noyer les Grecs, et leur mille Vaisseaux,

Mer, tu n'ouvriras pas des abîmes nouveaux?

Quoi, lorsque les chassant du Port qui les recèle,

L'Aulide aura vomi leur flotte criminelle,

Les Vents, les mêmes Vents si longtemps accusés,

Ne te couvriront pas de ces Vaisseaux brisés ? [1682-1688]

L'élargissement cosmologique du théâtre de la vengeance, comme la pluralisation hyperbolique des criminels, constitue une amplification caractéristique de l'épidictique, et qui culmine dans l'apostrophe au soleil liant monstruosité passée et présente par l'évocation de la funeste ascendance d'Agamemnon :

Et toi, Soleil, et toi, qui dans cette contrée

Reconnais l'héritier, et le vrai Fils d'Atrée,

Toi, qui n'osas du Père éclairer le Festin,

Recule, ils t'ont appris ce funeste chemin. [1689-1692]

Acte originel de la criminalité hors-norme des Atrides, le Festin monstrueux est le support d'une théâtralité paradigmatique, où le Soleil recule, en témoin effaré d'un spectacle de stupeur et d'horreur, selon l'esthétique qui régit la véhémence noire propre à la parole d'exécration et d'ostracisation. Or si la formule de l'anathème - si quis dixerit... anathema sit - demeurait encore à l'horizon de la conscience religieuse du $\mathrm{XVII}^{\mathrm{e}}$ siècle, la pratique de la parole d'exclusion et de condamnation a en revanche pratiquement disparu de nos cultures occidentales contemporaines. Aussi la véhémence cosmologique du discours de Clytemnestre résonne-t-elle étrangement dans nos consciences modernes : comme formidablement archaïque et décalée. Cette tirade imprécatrice revêt un caractère de transgression et d'excès qui l'isole et en accentue la théâtralité et la puissance d'effarement-la véhémence imprécatrice apparaissant aujourd'hui comme une parole incorrecte et asociale, provocante et dérangeante. D'où la difficulté à interpréter et recevoir aujourd'hui cette scène et les scènes raciniennes équivalentes d'imprécation (telle celle, déjà citée, de Jocaste dans $L a$ Thébaïde), à une époque où l'acteur ne possède plus le code déclamatoire propre à l'énoncé des grandes passions, et où le public n'est plus familier des apostrophes cosmologiques et de la forme noire de l'épidictique. Conjointe à l'effacement des codes oratoires de la scène classique, l'évolution des cultures et croyances a rendu problématique la restitution sur les plateaux contemporains de la théatralité si singulière de cette scène.

\section{Hypotypose fabulaire et performance scénique}

\section{L'Efficacité symbolique de l'imprécation}

31 L'apostrophe impérieuse aux éléments exacerbe la conjonction oxymorique de la forme jussive et de l'impuissance effective; contradiction inscrite dans la formulation négative du reproche : "Mer, tu n'ouvriras pas... », «Les Vents ne couvriront pas... ». Seul le Soleil reçoit un ordre direct-«Recule»-, mais cet ordre atteste 
paradoxalement de son impuissance à empêcher l'itération monstrueuse de l'infanticide. L'imprécation s'achève ainsi symboliquement sur la figure astrale d'un recul et d'une protestation aussi vaine que la gesticulation initiale de la reine.

Cependant, le retrait du soleil est aussi un dispositif spéculatif qui le fait regarder la scène; un regard qui est une reconnaissance, terme qui en poétique aristotélicienne désigne le moment aléthique au sein de la catastrophe ${ }^{21}$. Un méta-regard, par conséquent, qui explicite la fonction dramaturgique de la scène (celle-ci excluant toute forme dramatique de reconnaissance, en raison de son a-dramaticité). En revanche, la scène offre une forme métadramatique de reconnaissance par la mise en lumière de la double monstruosité des criminels: celle d'Ériphile, fille de Mégère, et celle d'Agamemnon, fils d'Atrée. Une reconnaissance métadramatique qui fonctionne selon une double perspective :

a. Une perspective éthique et cognitive, incarnée par le regard surplombant de l'astre solaire, regard qui, dans la répétition de l'infanticide mythique, reconnaît, c'est-à-dire révèle, l'essence morale du fils des Atrides. La scène construit un surplomb cognitif focalisé sur les manifestations de la monstruosité, apparentant la théâtralité de la scène à un dispositif philosophique aléthique.

b. Une perspective dramaturgique générale, qui articule le drame à la fable en régissant la relation entre la représentation et la mémoire du spectateur. En effet, le discours épidictique d'accusation et de malédiction met en place une préfiguration symbolique des châtiments à venir : 1) le suicide d'Ériphile qui, dans le temps dramatique concomitant ou immédiatement postérieur à l'imprécation, accomplit le vœu de mort formulé par Clytemnestre; 2) l'assassinat d'Agamemnon qui, dans la temporalité fabulaire, accomplira à long terme le vœu de vengeance de la reine.

L'imprécation revêt ainsi une valeur prédictive et met en place une causalité métadramatique qui redonne une efficacité symbolique à la forme noire du discours épidictique.

Or cette efficacité symbolique procède non de la rhétorique intrascénique, dépourvue d'efficacité dramatique, mais de la rhétorique extrascénique. Préfiguration symbolique du destin des protagonistes, l'imprécation s'adresse directement au spectateur en sollicitant sa mémoire de la fable homérique. C'est pourquoi l'apostrophe de Clytemnestre met en jeu l'hypotypose, support de l'imaginaire épique. En invoquant le pouvoir destructeur de la Mer et des Vents, Clytemnestre peint en effet dispersion et anéantissement de la flotte grecque selon le paradigme homérique; mais elle en inverse la chronologie puisqu'elle envisage cette catastrophe vengeresse au départ pour Troie et non au retour. Réécrivant ainsi l'Iliade sur le patron de l'odyssée, la tirade de Clytemnestre transforme la matière épique en tragédie.

L'hypotypose de la flotte dispersée et détruite possède une double fonction macro- et micro-structurelle: 1) dans l'économie dramaturgique générale de la pièce, l'hypotypose imprécatrice du naufrage déconstruit l'hypotypose triomphale peinte par Ulysse à l'initiale du drame ${ }^{22}$ : cette double structure d'hypotypose forme un diptyque antithétique qui encadre l'action du drame et l'insère dans la longue durée de la fable homérique. 2) Ce rapport à la fable détermine à son tour, dans le fonctionnement de l'imprécation, un jeu temporel subtil reposant sur la mémoire culturelle du spectateur. En effet, si dans la perspective de la temporalité scénique, l'imprécation prédit le futur immédiat (le suicide d'Ériphile) et le futur à long terme (l'assassinat d'Agamemnon), ces mêmes vœux appréhendés dans la perspective extrascénique d'un spectateur informé 
de la fable homérique, constituent un futur antérieur d'événements qu'il reconnaît dans le temps même où Clytemnestre les souhaite ${ }^{23}$. Une complicité mémorielle détermine la réception de la tirade par le spectateur en raison de la familiarité de l'imaginaire qu'elle déploie et suscite ${ }^{24}$.

Appréhendée dans son « efficacité » symbolique, l'imprécation fonctionne alors selon le paradigme aristotélicien de la statue de Mitys $^{25}$ : ce que la tirade n'accomplit pas au plan du drame, elle l'accomplit au plan extrascénique de la fable. L'imprécation véhémente rétablit au plan symbolique un principe de justice bafoué par la situation dramatique.

\section{Symbole et performance : le double statut du coup de tonnerre}

Par ce jeu symbolique, la logique imageante du discours semble au plus lointain du plateau qui montrait le corps entravé et tourmenté de la Reine ; la tentation est grande alors de qualifier d'abstraite cette scène investie par l'imaginaire de la parole et l'interaction culturelle du dramaturge et de son public (du moins sa partie cultivée et lettrée). Une telle lecture est possible, et elle est fondée, précisément, en tant que lecture du texte. Mais elle est contredite par l'analyse de la représentation - si l'on veut bien déchiffrer les derniers vers de la tirade à la lumière de la performance scénique :

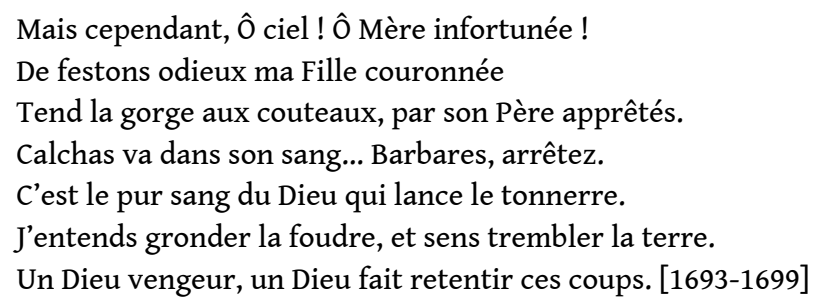

L'apostrophe aux Éléments fait place à une interpellation des Grecs oxymoriquement qualifiés de «barbares », écho aux " perfides » de l'ouverture, et interpellation dont la valeur déictique est actualisée par la présence des gardes sur scène. Le temps de l'énonciation renoue donc avec le temps de l'énoncé (conformément au sens littéral et classique de «cependant »). L'hypotypose, qui précédemment concernait le futur, réfère désormais au temps présent et superpose espace scénique et hors-scène-où s'accomplit le sacrifice ${ }^{26}$. Ce sacrifice, toutefois, est encore une vision de Clytemnestre, effet de l'effroi et de la terreur qui la transportent. Cette image d'Iphigénie sacrifiée (que Racine s'est interdit de réaliser scéniquement, et que le dénouement, ultimement, esquive) procède de la logique de l'imprécation furieuse, fondée sur l'expansion de l'imaginaire théâtral ; et dans cette perspective du signe véhément, les grondements et tremblements signalés par Clytemnestre, s'intègrent parfaitement dans le cadre cosmologique de la malédiction. La logique du discours véhément n'est donc pas rompue, comme le souligne Georges Forestier, en rappelant le lien mythologique entre la foudre et l'ascendance d'Iphigénie :

Magnifique passage où l'on pense que, par association d'idées - Iphigénie, on le sait, descend par son père et sa mère $d u$ " Dieu qui lance le tonnerre " (Zeus) -, Clytemnestre croit entendre la foudre. On ne comprendra que deux scènes plus loin, à la fin du récit d'Ulysse, qu'elle entendait effectivement le coup de tonnerre qui suivit la mort d'Ériphile (v. 1778) ${ }^{27}$.

Cependant, ce commentaire littéraire érudit met aussi en évidence la réalité dramatique du coup de tonnerre qui ponctue la mort d'Ériphile et la résolution de la crise tragique. Clytemnestre n'est donc pas frappée d'hallucination sensorielle, et sa 
vision horrifiée du sacrifice de sa fille coïncide avec un coup de tonnerre effectif - et qui constitue le point de convergence du discours véhément et de la violence du drame. Aussi l'analyse de Georges Forestier demeure-t-elle dans la logique d'une lecture du texte où effectivement « on ne comprendra que deux scènes plus loin » le sens exact de ce tonnerre. Mais pour un spectateur, au même titre que pour Clytemnestre, ce coup de tonnerre correspond à un élément concret de performance scénique : un coup de tonnerre - ou le bruitage qui en tient lieu sur le plateau - retentit effectivement dans le moment exact où Clytemnestre déclare «J'entends gronder la foudre et sens vibrer la terre ». Dans la logique du spectacle, il ne faut donc pas dire "Clytemnestre croit entendre le tonnerre ", mais Clytemnestre "entend le tonnerre». La suspension au centre du vers 1696 ne marque pas l'hésitation de Clytemnestre, mais littéralement sa stupeur, sa sidération, à l'instant précis où résonne un tonnerre qui amuït l'acteur. Si donc le lecteur doit attendre deux scènes pour comprendre la réalité et la signification du tonnerre perçu par Clytemnestre, le spectateur en revanche entend hic et nunc, un très réel coup de tonnerre (de théâtre ${ }^{28}$ ).

La véhémence oratoire renoue ainsi avec la performance scénique. Contemporaine et concurrente du spectaculaire de la tragédie en musique, Iphigénie, tragédie en paroles, augmente son efficacité spectaculaire par la conjonction de l'imaginaire discursif et de la performance scénique. Le spectacle des passions est en parfaite coïncidence avec le spectacle tout court : dira-t-on encore que la scène racinienne est abstraite ?

Quelles leçons tirer de cette interaction de la rhétoricité et de la théâtralité dans la dramaturgie racinienne?

1. L'a-dramaticité offre un exceptionnel plateau à la monstration des passions et promeut les genres judiciaire et épidictique, conséquence de l'aporie de l'action et de la délibération.

2. Le regard du spectateur se focalise ainsi sur l'actio corporelle et oratoire du protagoniste, privé de tout agir. Agitation et gesticulation actoriales présentent la figure allégorique du corps et de l'âme souffrants, articulation d'un envers corporel et d'un avers spirituel. Le trouble désigne alors simultanément l'espace scénique enfermant le personnage et l'espace psychique de sa souffrance.

3. La construction de la fureur procède d'une structure dialogique graduée: d'abord déterminée par l'intervention provocatrice d'Aegine, elle se poursuit dans le schème dialogique par lequel Clytemnestre apostrophe in absentia les Éléments. La théâtralité de la fureur procède de ce dispositif discursif et rhétorique qui détermine l'attente du spectateur dont le regard est convié à la monstration des grandes passions, dans une situation analogique du suave mari magno.

4. Le discours d'imprécation va du genre judiciaire au genre épidictique ; la figure des Érinyes, déesses de la fureur et de la vengeance, transformant l'accusation de la seule Ériphile en un désir de vengeance élargi aux dimensions du cosmos antique. L'apostrophe épidictique convoque alors l'hypotypose où l'intentionnalité vengeresse subvertit l'imaginaire épique, instituant l'astre solaire en spectateur d'un théâtre de l'horreur et de la monstruosité.

5. Jouant de la mémoire du spectateur dans la double temporalité des vengeances à venir (dans la chrono-logique du drame) et des vengeances accomplies (dans la mémoire culturelle du spectateur), l'imprécation de Clytemnestre retrouve une efficacité symbolique en préfigurant les supplices qui scelleront le destin d'Ériphile et d'Agamemnon. Une causalité métadramatique compense ainsi l'aporie dramatique et délibérative de la scène par le rétablissement imaginaire et symbolique du principe de justice.

6. Au paroxysme du discours véhément, un coup de tonnerre met en coïncidence cette causalité symbolique et la performance scénique qui fait effectivement entendre le tonnerre 
sur le plateau, conjuguant spectaculaire scénique et véhémence oratoire, imaginaire discursif et (re) présentation scénique.

42 Chemin faisant, l'analyse invalide quelques lieux communs de la critique racinienne. La dramaturgie racinienne n'est pas toute entière régie par la nécessité dramatique aristotélicienne. La mise en panne de l'action et de la délibération offre un espace de monstration des passions qui promeut rhétoricité, théâtralité et métathéâtralité. Corrélativement, le genre oratoire recteur de la tragédie est le genre délibératif et non le genre judiciaire, ce que la scène illustre a contrario. De même, le corps actorial racinien n'est nullement une abstraction, mais bien un objet scénique (au sens classique) constamment pris en charge par les didascalies internes d'un texte dont l'horizon est la représentation et l'incarnation. La scène racinienne n'est pas davantage abstraite, mais le lieu de la performance, que manifeste le coup de tonnerre final. Ainsi la rhétorique racinienne est-elle une rhétorique authentiquement théâtrale déterminée par l'horizon pragmatique de son actualisation scénique. En contrepoint du processus de littérarisation qu'a connu la rhétorique à maintes reprises dans son histoire occidentale, notamment moderne, le théâtre constitue un évident contrepoint où l' actio, fonctionnellement absente d'une rhétorique littérarisée, est au contraire déterminante et omniprésente.

L'étude de ce cas singulier d'a-dramaticité racinienne a révélé l'interaction fonctionnelle de la théâtralité et de la rhétoricité dans la double monstration du corps et de la parole en véhémence. L'émergence de cette modalité oratoire liée à l'intensité passionnelle et émotionnelle est suscitée par l'aporie dramatique : la véhémence est en ce sens la manifestation d'une fureur vaine ; ce qui la met à distance de la violence et l'apparente au simulacre. Cette définition doit cependant être amendée lorsqu'on adopte la perspective métadramatique où l'imprécation est investie d'une fonction prédictive, prophétique ou magique par laquelle l'expression du désir de vengeance préfigure son accomplissement. Au regard de la fable, la parole de Clytemnestre ne se réduit pas à un wishful thinking, et cet efficace au lointain de la scène et du drame renforce le caractère inquiétant de l'épidictique noir... C'est dire aussi qu'en tragédie aucune énonciation n'est vraiment stérile et que jouer de la puissance de la parole n'est jamais un acte gratuit. Dans la progression de l'imprécation, la rhétoricité comprise comme la pression du langage sur le réel, prend ainsi le pas sur la théâtralité vaine du simulacre. C'est dire encore que le spectateur ne sort pas totalement indemne du spectacle de monstration des passions. D'une part, nous l'avons vu, parce que celui-ci sollicite sa mémoire culturelle pour mettre en place une temporalité feuilletée par laquelle l'imprécation retrouve une efficacité symbolique métadramatique. D'autre part et surtout parce que la dramaturgie, en liant in fine l'imaginaire vengeur à la réalité de la performance scénique, littéralement, surprend le spectateur dans le temps même où le tonnerre effare Clytemnestre. Mais ce tonnerre scénique fait plus encore, car il masque en son évidence une ambiguïté qui est la marque de la sophistique qui imprègne la dramaturgie et l'esthétique raciniennes. Car si la réalité du coup de tonnerre s'impose sur la scène, sa confrontation à l'imaginaire de l'imprécation suscite chez Clytemnestre, et a fortiori chez le spectateur, une herméneutique erronée. Persuadée que sa fille vient d'être sacrifiée, la reine interprète le tonnerre comme le signe de la vengeance par les dieux de cette mort («Un Dieu vengeur, un Dieu fait retentir ces coups »); plus précisément encore, elle fait du sang versé d'Iphigénie la source de ce coup de tonnerre: "C'est le pur sang du Dieu qui lance le tonnerre ». Ce 
faisant, Racine met en hypotypose une image du sacrifice que son drame ne met pas en scène. L'image et l'interprétation de Clytemnestre sont de fait démenties par le récit d'Ulysse : le sang qui coule effectivement est celui d'Ériphile; de sorte que le coup de tonnerre n'annonce pas une vengeance à venir, mais ponctue son accomplissement. Ainsi l'hypotypose sacrificielle envisagée par Clytemnestre se révèle-t-elle fallacieuse... Au cœur de la tragédie classique et au point d'évidence de la performance scénique, l'ambiguïté sémantique et éthique du coup de tonnerre installe un singulier trompel'œil où un sacrifice peut en cacher un autre. Racine joue ainsi paradoxalement de l'équivocité de l'évidence du signe : plus celui-ci est transparent, plus il est illisible. Une virtuosité sophistique que Pierre Fontanier avait bien discerné dans l'usage racinien des figures :

Il les emploie si naturellement, il les fond tellement dans sa phrase, qu'elles s'y cachent en quelque sorte, et qu'il faut souvent de la réflexion pour les y apercevoir, lors même qu'elles sont le plus hardies ${ }^{29}$.

Tant il est vrai que chez Racine, ce sont moins les dieux que le sens qui se cache dans l'apparente simplicité des signes du discours. Un sens qui ne peut se révéler sans la performance scénique pour laquelle le dramaturge a conçu ces signes, entre évidence et équivoque, trouble et luminosité.

\section{NOTES}

1. Une première version de ce travail a paru sous le titre « Rhétoricité et théâtralité raciniennes : Réflexions sur une scène inutile (Iphigénie, V, 4) », dans Changing Perspectives. Studies on Racine in Honor of John Campbell, R.W.Tobin \& A. J. Kennedy éd., Charlottesville, Rookwood Press, 2012, p. 51-63. Le présent texte prolonge le précédent notamment dans la définition de la théâtralité et dans ses conclusions.

2. Sur pathos et grand style, voir A. Michel, Les rapports de la philosophie et de la rhétorique dans l'œuvre de Cicéron, P.U.F., 1960, rééd. Peeters, 2003.

3. "Théâtre de Baudelaire », dans Essais critiques, Paris, Le Seuil (« Points »), 1981 (1954), p. 41. Pour un recensement des différentes acceptions du terme, voir E. Eigemann, Méthodes et problèmes. Le Mode dramatique, U. de Genève, 2003 (http://www.unige.ch/lettres).

4. Voir S. Chaouche, L'Art du comédien. Déclamation et jeu scénique en France à l'âge classique (1629-1680), Paris, Champion, 2001. Le souci de la dynamique actoriale avait déterminé G. Forestier à retenir en 1999 les éditions originales de Racine (graphie et ponctuation reflétant la prononciation scénique). Perspective abandonnée dans sa récente édition de Molière (G. Forestier \& C. Bourqui éd., Gallimard, «Bibliothèque de la Pléiade », 2010), ordonnée par l'histoire du théâtre imprimé. Nous restons fidèle à la première perspective, arrimée à la performance théâtrale, et citons selon cette édition (Racine, Euvres complètes, Théâtre-Poésie, G. Forestier éd., Gallimard, « Bibliothèque de la Pléiade », t. 1, 1999).

5. Poétique, ch.6,50a15, R. Dupont-Roc \& J. Lallot éd., Paris, Le Seuil, 1980 : «J'appelle ici "histoire" [muthos] le système des faits" (50a5); «le plus important de ces éléments est l'agencement des faits en action. » 
6. Ibid., 50a19-23 : «[Les hommes] n'agissent pas pour représenter des caractères, mais c'est au travers de leurs actions que se dessinent leurs caractères. De sorte que les faits et l'histoire sont bien le but visé par la tragédie, et le but est le plus important de tout. "

7. On trouvera chez Brecht l'énoncé de cette distinction entre le pathos de la chose vue et le logos critique du compte-rendu de la chose vue («la scène de rue comme modèle du théâtre épique ", dans L'Achat du cuivre, éd. l'Arche, p. 520 sq.). Brecht distingue le narrateur de l'accident du spectateur par l'ascèse émotionnelle que le premier exerce sur lui-même afin de donner à son auditoire un compte-rendu permettant à celui-ci de former sa propre opinion sans être soumis au jeu aliénant de la sympathie émotionnelle. Voir G. Declercq, «Pathos et théâtralité. Pour une économie cognitive des passions ", dans Émotions et discours. L'Usage des passions dans la langue, M. Rinn éd., Presses de l'université de Rennes, avril 2008, p. 219-245.

8. Rhétorique, I, 1, 1355 b, 11, trad. Médéric Dufour, Les Belles Lettres, 1932 : «Sa fonction propre n'est pas de persuader, mais de voir les moyens de persuader que comporte chaque sujet. »

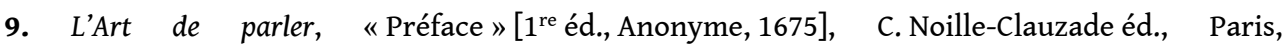
H. Champion, 1998, p. 439.

10. C'est ainsi qu'il faut comprendre l'expression de la préface de Bérénice qui désigne l'effet esthétique global exercé par la représentation de l'œuvre: "tout s'y ressent d'une tristesse majestueuse ». Voir G. Declercq, «Représenter la passion : la sobriété racinienne», Littératures Classiques, 11, 1989, p. 69-93.

11. Scène exemplaire mais non pas unique: on peut en lire l'esquisse dans le monologue de Jocaste (La Thébaïde, III, 2), où la Reine, en des termes singulièrement semblables à ceux de Clytemnestre, fait querelle aux Dieux de leur cruauté, et, usant pareillement des genres judiciaire et épidictique, recourt au topos des supplices infernaux (677-678) : « Me feront-ils souffrir tant de cruels trépas, / Sans jamais au tombeau précipiter mes pas... "; voir encore 685-686. L'analogie est encore plus globale avec l'apostrophe in absentia par laquelle Jocaste déploie une rhétoricité fondée sur la monstration d'une fureur impuissante, en marge d'une action dramatique qui s'accomplit hors-scène. Notre objet d'étude est précisément cette forme particulière de théâtralité qui conjoint véhémence oratoire et performance scénique, imaginaire discursif et image du corps actorial.

12. Discours du poème dramatique, 1660 , dans Euvres complètes, G. Couton éd., Paris, Gallimard («Bibliothèque de la Pléiade »), 1980-87, t. III, p. 124 : "Sa dignité demande quelques grands malheurs d'État [...]. S'il ne s'y rencontre point de péril de vie, de pertes d'États, ou de bannissement, je ne pense pas qu'il ait droit de prendre un nom plus relevé que celui de comédie. »

13. Cf. d'Aubignac: "Car là parler c'est agir » (La Pratique du théâtre, 1669, II, ch.4, H. Baby éd., Paris, H. Champion, 2001, p. 407).

14. Ce lien essentiel entre la rhétorique délibérative et la dramaticité de la tragédie invalide une doxa aussi erronée que tenace qui présente le genre judiciaire comme le genre oratoire recteur de la tragédie. Il n'en est rien, d'une part en raison de l'impossibilité de trouver un personnagejuge dans la tragédie, genre politique où tout personnage est intéressé à l'action, alors que la position de juge suppose une attitude arbitrale et neutre; d'autre part en raison d'un amalgame impropre entre la polémicité des dialogues en tragédie et la rhétorique judiciaire. Les scènes de confrontation en tragédie sont des scènes d'opposition politique - au sens de conflit de pouvoir -, elles n'ont que l'apparence de procès, et les arguments, même juridiques, sont toujours déterminés par des enjeux délibératifs. Sur cette question, voir G. Declercq, "L'identification des genres oratoires en tragédie française", dans Theatrum mundi. Studies in Honor of Ronald W. Tobin, C. Carlin \& K. Wine éd., Charlottesville, Rookwood Press, 2003, p. 230-238. 15. Le pendant de cette impuissance royale est l'image d'Agamemnon se couvrant le visage devant le sacrifice de sa fille, peinture antique (perdue) de Timanthe, glosée par Quintilien (Institution oratoire, II, 13, 13) et transposée par Racine (Iphigénie, V, 5, 1698-1700). 
16. Sur cette distinction, voir A.Greimas et J.Fontanille, Sémiotique des passions, Paris, Le Seuil, 1991, p. 245.

17. Ainsi faisait Sganarelle, dans la mise en scène de Dom Juan (Avignon, 1978), protestataire impuissant, tournant autour de son maître occupé à séduire Charlotte.

18. Marc Fumaroli rappelle cette imprégnation allégorique du théâtre à propos de Phèdre («De Médée à Phèdre: naissance et mise à mort de la tragédie cornélienne ", Héros et orateurs, Genève, Droz, 1990, p. 508) : "Dès son apparition sur scène à l'acte I, celle-ci est orchestrée par une série de métaphores du jour, de la lumière sur fond nocturne. [...] Autant d'images classiques renvoyant au théâtre comme dévoilement dans la lumière, et l'identifiant au passage solennel du personnage, de la nuit et de l'absence du personnage à son éclatante apparition sur la scène illuminée. Avec Phèdre qui s'avance sur la scène, c'est le théâtre qui dans ce mouvement se résume et se réfléchit. »

19. Anne Surgers a mis en évidence cette bivalence dans les arts de représentation renaissants et classiques (dans Et que dit ce silence. Trésor des figures de rhétorique du visible, Paris, Presses de la Sorbonne-nouvelle, 2007, p. 138), notamment à propos d'un portrait d'Élisabeth d'Angleterre, ou Portrait à l'arc-en-ciel, attribué au peintre Gheeraerts (circa 1600-1613) : "Grâce à la multiplication de signes polysémiques, le Portrait à l'arc-en-ciel exprime et manifeste les "deux corps" de la royauté, corps physique et temporel de la reine / corps mystique et glorieux du pouvoir royal. C'est un des éléments qui ont contribué à la construction du pouvoir royal élisabéthain, au même titre que le théâtre. »

20. Dialogue entre Socrate et le peintre Parrhésios, rapporté par Xénophon (Mémorables, III, 10, 11) et glosé par Pascal Quignard (Le Sexe et l'effroi, Paris, Gallimard, 1994, p. 53-54). Saisi par le regard du peintre, le visage est l'expression morale de l'âme (to tès psuchès èthos), avers physique et envers psychique. Sur ces questions, voir G. Declercq, «La Mémoire de l'image. L'allégorie racinienne entre littérarité et théâtralité ", dans L'imaginaire théâtral, J. de Guardia, V. Locher, Fr. Toudoire-Surlapierre dir., Dijon, Presses de l'Université de Bourgogne, à paraitre.

21. « Toi, qui dans cette contrée / Reconnaît l'héritier, et le vrai Fils d'Atrée » (v. 1689-1690). Sur la reconnaissance, voir T. Cave, Recognitions. A study in Poetics, Oxford, Clarendon, 1988, rééd. 1998. 22. "Voyez tout l'Hellespont blanchissant sous nos rames, / Et la perfide Troie abandonnée aux flammes...» (I, 5, 381-388). Sur cette figure, voir G. Declercq, «À l'école de Quintilien. L'hypotypose dans les tragédies de Racine", dans la revue Op.cit., Université de Pau, 5, 1995, p. 73-88.

23. Même jeu temporel et métadramatique dans l'hypotypose d'Ulysse, « Et ce triomphe heureux, qui s'en va devenir / L'éternel entretien des siècles à venir. » (I, 5, v. 387-388).

24. Familiarité fabulaire qui régit l'évocation d'Agamemnon et de son destin funeste; le destin d'Ériphile, de moindre notoriété (quoi qu'en dise Racine dans sa Préface en invoquant Stésichore), s'inscrira dans le cadre du drame, lorsque l'imaginaire du discours de Clytemnestre aura rejoint présent et performance scéniques. (Voir ci-dessous notre analyse de l'interaction de l'imprécation et du dénouement.)

25. Le châtiment d'Ériphile notamment «satisfait " le désir de justice du spectateur selon un principe d'économie éthique qui, tel que l'expose Aristote (Poétique, IX, 52 a 1-11), régit l'épisode de la statue de Mitys qui, dans sa chute, tue l'assassin de ce dernier: l'événement dramatique correspond ainsi à une nécessité symbolique.

26. C'est la seconde fois que Clytemnestre (se) représente le sacrifice de sa fille, une première fois évoqué pour en accuser Agamemnon: «Un Prêtre environné d'une foule cruelle, / Portera sur ma Fille une main criminelle?» (IV, 5, v. 1301-1302). La seconde occurrence, en clôture de l'imprécation, est donc littéralement l'actualisation de cette première hypotypose prospective, judiciaire et conjuratoire.

27. T. 1, p. 760, note 2. Cf. Ulysse : « À peine son sang coule et fait rougir la terre ; / Les Dieux font sur l'Autel entendre le tonnerre » (V, dernière scène, 1777-1778). 
28. De même, pour l'acteur qui lit le texte pour le jouer, le rapprochement du témoignage auditif de Clytemnestre et la mention du tonnerre dans le récit d'Ulysse n'ont pas valeur explicative mais prescriptive : un coup de tonnerre, grondant et vibrant, doit ponctuer et suspendre la fin du discours d'imprécation.

29. Les Figures $d u$ discours [1821-1827], Paris, Flammarion, «Introduction » par G. Genette, 1968, p. 183.

\section{AUTEUR}

\section{GILLES DECLERCQ}

Institut d'Études Théâtrales de la Sorbonne nouvelle 